\title{
k-Scanning: An Alternative to 4D-STEM
}

\author{
Andrew Barnum ${ }^{1 *}$ \\ 1. Thermo-Fisher Scientific, Hillsboro, OR, USA. \\ * Corresponding author: andrew.barnum@thermofisher.com
}

Following advancements in high speed pixelated detector technologies, there has been an increasing interest in the use of scanning nano-diffraction imaging to collect 4D-STEM datasets for computational imaging experiments [1]. Detailed structural information from a specimen can be extracted by combining real space scanning with the detection of diffraction patterns in reciprocal $k$-space. Unfortunately, both the specialized detectors and quantity of data produced during 4D-STEM imaging poses a barrier to routine use. An alternative methodology is possible due to the reciprocity principle, since a real space scan with a detector covering $k$-space in STEM is equivalent to an image-beam tilt series through $k$-space with a detector covering real space. Scanning through reciprocal space using the tilt coils can be called " $k$-scanning," since the beam is not physically scanned across the surface of the specimen, and the resulting four-dimensional matrix referred to as a "4D-TEM image."

While this technique has been discussed previously in literature [2] and seen some limited use experimentally, it is only relatively recently that the combination of camera frame rate, computer storage and processing have enabled 4D-TEM imaging to become a practical technique for routine experiments. As a four-dimensional imaging method, the number of images acquired increases quadratically, making the datasets acquired in either mode consume several gigabytes per acquisition. Fortunately, the proliferation of data processing methods designed for handling 4D-STEM images can be applied directly to 4D-TEM images by transposing the matrix from $\left(\mathrm{k}_{\mathrm{x}}, \mathrm{k}_{\mathrm{y}}, \mathrm{x}, \mathrm{y}\right)$ to $\left(\mathrm{x}, \mathrm{y}, \mathrm{k}_{\mathrm{x}}, \mathrm{k}_{\mathrm{y}}\right)$. The $k$-scanning process also provides an interesting pedagogical benefit in assisting non-experts understand higherdimensional analytical imaging techniques [3]. The frequency cut-off caused by the objective aperture through the exclusion of diffracted beams, and the "formation" of a Ronchigram or CBED pattern observed as different combinations of the central and diffracted beams scanned across the aperture.

Mobile hydrocarbons on the surface of a specimen deposit as carbon contamination on contact with the edge of the electron beam. Current dwell times used with existing fast pixelated detectors range from the hundreds of microseconds to several milliseconds, causing significant concerns regarding contamination build up, beam damage, and drift. Under parallel illumination, the beam edge can be shifted well outside of the imaging area, placing the regions of maximum contamination and damage off of the region of interest. Drift is corrected more easily since each frame provides a real space image that can be crosscorrelated with neighbouring frames for alignment. With these considerations, $k$-scanning may be preferable for some experiments over 4D-STEM

Image-beam tilting refers to the use of the image tilt coils below the specimen plane to compensate for any tilt introduced above the specimen using the beam tilt coils. The objective aperture sets the effective convergence angle for the resulting pattern, while the angular range set in the $k$-scan sets the effective collection angle. As the beam is tilted, the diffraction pattern is swept across the objective aperture, permitting only sub-regions of the diffraction pattern to contribute to the image at each tilt value. Images were acquired on a Titan Metrios using the Ceta camera, and acquisition-tilt synchronization was completed using TEMScripting. 


\section{References:}

[1] MW Tate et al., 2016. Microscopy and Microanalysis 22(1) (2016), p. 237.

[2] T Plamann and JM Rodenburg, 1998. Acta Crystallographica Section A 54(1) (1998), p. 61.

[3] JCH Spence in "High-resolution Electron Microscopy", $4^{\text {th }}$ Ed, (Oxford University Press, Oxford).

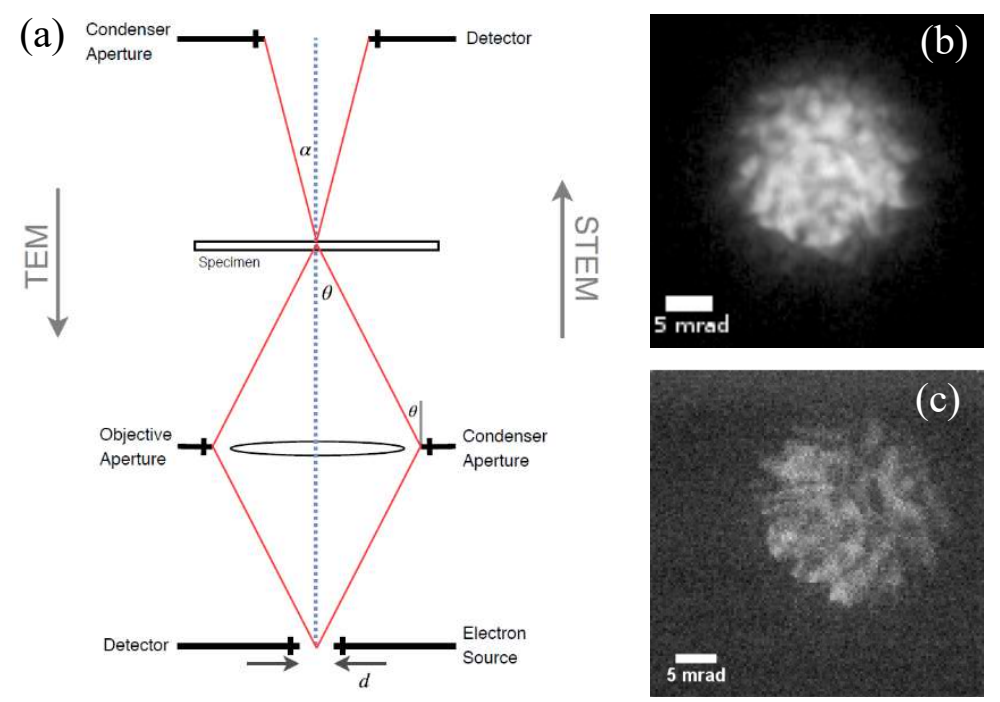

Figure 1. (a) The reciprocity principle in optics permits the reversal of an optical system to generate equivalent datasets for both 4D-STEM and 4D-TEM (b) STEM CBED pattern (c) $k$-scan patterns collected under using a $40 \mathrm{um}$ objective aperture to match the STEM convergence angle of approximately $12 \mathrm{mrad}$.
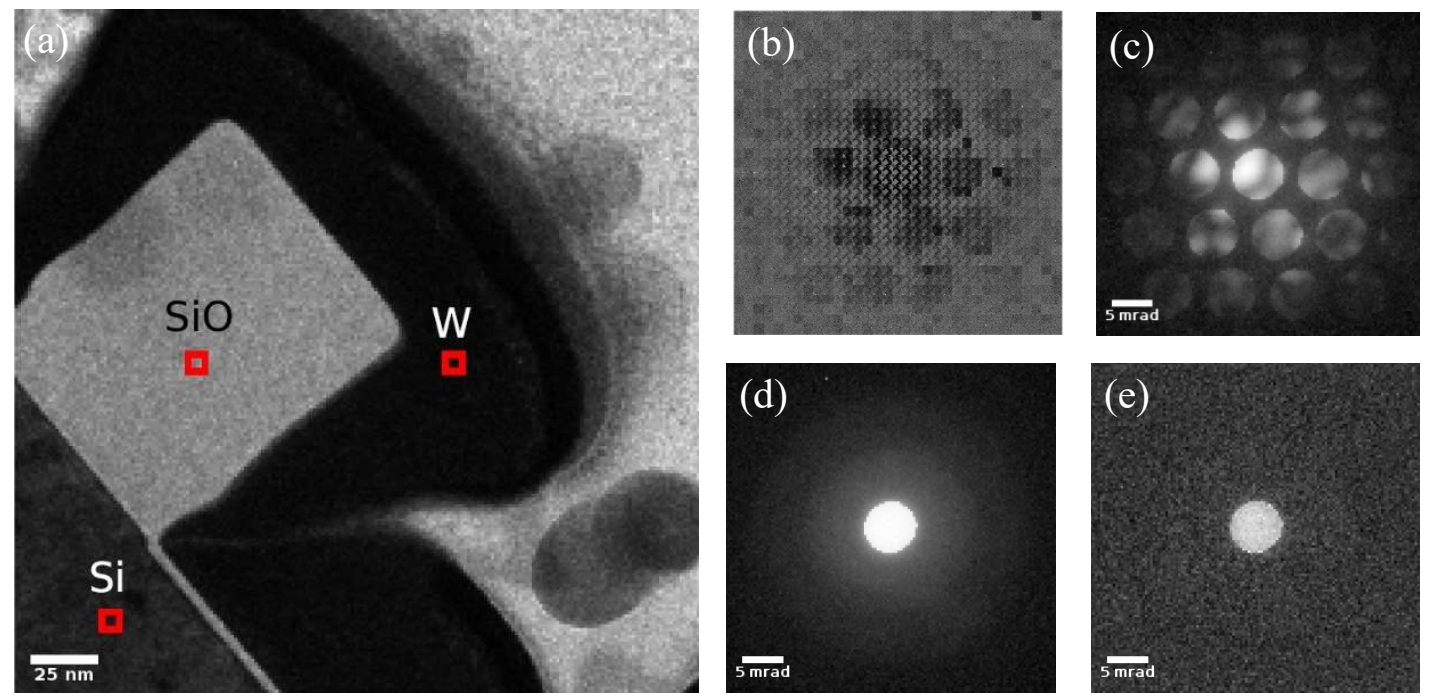

Figure 2. (a) 4D-TEM dataset collected on a Metrocal specimen as a reference semiconductor structure. A $10 \mathrm{um}$ aperture yielded the equivalent of a $3 \mathrm{mrad}$ convergence semi-angle. As seen in (b), a $32 \times 32$ point $k$-scan illustrates the generation of the diffraction pattern when tilting TEM images. (c) $128 \times 128$ point $k$-scan on silicon (d) same $k$-scan over silicon dioxide (e) $k$-scan over the tungsten layer. 